\title{
SIMPLICITY AND TYPICAL RANK RESULTS FOR THREE-WAY ARRAYS
}

\author{
JOS M.F. TEN BERGE \\ UNIVERSITY OF GRONINGEN
}

\begin{abstract}
Matrices can be diagonalized by singular vectors or, when they are symmetric, by eigenvectors. Pairs of square matrices often admit simultaneous diagonalization, and always admit block wise simultaneous diagonalization. Generalizing these possibilities to more than two (non-square) matrices leads to methods of simplifying three-way arrays by nonsingular transformations. Such transformations have direct applications in Tucker PCA for three-way arrays, where transforming the core array to simplicity is allowed without loss of fit. Simplifying arrays also facilitates the study of array rank. The typical rank of a three-way array is the smallest number of rank-one arrays that have the array as their sum, when the array is generated by random sampling from a continuous distribution. In some applications, the core array of Tucker PCA is constrained to have a vast majority of zero elements. Both simplicity and typical rank results can be applied to distinguish constrained Tucker PCA models from tautologies. An update of typical rank results over the real number field is given in the form of two tables.
\end{abstract}

Key words: tensor decomposition, tensor rank, typical rank, sparse arrays, Candecomp, Parafac, Tucker component analysis.

Three-way data arise when a complete set of measures is obtained for a set of individuals or objects on a set of variables, in a set of circumstances or conditions. For instance, when $I$ subjects are evaluated on $J$ tests on $K$ different occasions, the data consist of an $I \times J \times K$ three-way array. The array can be cut up into slices: there are $K$ frontal slices of order $I \times J, J$ lateral slices of order $I \times K$, and $I$ horizontal slices of order $J \times K$.

Tucker (1966), Carroll and Chang (1970), and Harshman (1970) have proposed generalizations of principal component analysis (PCA) for the analysis of three-way arrays. For a long time, these methods were well known in psychometrics, but did not receive much attention elsewhere. A few decades ago, however, chemometricians discovered that these methods are particularly useful for their own purposes of data analysis. Nowadays, three-way component analysis is at the heart of what is called tensor decomposition analysis, which has applications in various areas such as signal processing, numerical linear algebra, computer vision, numerical analysis, data mining, graph analysis, neuroscience, and others (Kolda \& Brader, 2009). Although tensor decomposition analysis covers multiway arrays beyond the three-way level, only three-way component analysis will be considered in the present paper.

Three-way component analysis poses various mathematical problems that have been studied extensively. Among these are the uniqueness properties of three-way decompositions and the transformational freedom that exists when they are absent, the possibilities of transforming arrays to simple forms (with as many vanishing elements as possible, that is), and the rank of three-way arrays. The present paper is focused on the latter two topics. The purpose is to explain simplifying transformations for three-way arrays and to give an overview of typical rank results for three-way arrays (over the real number field).

The organization of this paper is as follows. After reviewing Candecomp, Parafac, and Tucker three-way PCA, it will be explained how problems of identification and triviality may

This research was done jointly with Henk Kiers, Roberto Rocci, Alwin Stegeman, and Jorge Tendeiro. The author is obliged to Henk Kiers and Mohammed Bennani Dosse for helpful comments.

Requests for reprints should be sent to Jos M.F. ten Berge, University of Groningen, Groningen, The Netherlands. E-mail: j.m.f.ten.berge@ rug.nl 
arise in the context of constrained Tucker component analysis. These problems can sometimes be handled by using maximal simplicity results and typical rank results. An overview of the latter results will be given.

\section{Candecomp/Parafac}

Carroll and Chang (1970) and Harshman (1970) independently rediscovered a method previously proposed by Hitchcock (1927a), and called it Candecomp and Parafac, respectively. We shall use its acronym $C P$. Let $\underline{\mathbf{X}}$ be a three-way array holding frontal slices $\mathbf{X}_{1}, \ldots, \mathbf{X}_{K}$ of order $I \times J$. For any fixed number of components $r, \mathrm{CP}$ yields component matrices $\mathbf{A}, \mathbf{B}$ and $\mathbf{C}$, with $r$ columns, minimizing $\sum \operatorname{tr}\left(\mathbf{E}_{k}^{\prime} \mathbf{E}_{k}\right)$ in the decomposition

$$
\mathbf{X}_{k}=\mathbf{A C}_{k} \mathbf{B}^{\prime}+\mathbf{E}_{k}
$$

where $\mathbf{C}_{k}$ is the diagonal matrix holding the elements of row $k$ of $\mathbf{C}$ in the diagonal, $k=1, \ldots, K$.

Under relatively mild conditions, $\mathrm{CP}$ has the property of essential uniqueness. This means that the columns of $\mathbf{A}, \mathbf{B}$ and $\mathbf{C}$ are determined up to rescaling and joint permutations. First, rescaling columns of $\mathbf{A}$ ( or $\mathbf{B}$ or $\mathbf{C}$ ) by a diagonal matrix $\mathbf{L}$ is allowed, provided that the inverse of $\mathbf{L}$ is accounted for elsewhere. For instance, when slice $\mathbf{X}_{k}$ is decomposed as $\mathbf{X}_{k}=\mathbf{A} \mathbf{C}_{k} \mathbf{B}^{\prime}+\mathbf{E}_{k}$, $k=1, \ldots, K$, the fitted parts $\mathbf{A C}_{k} \mathbf{B}^{\prime}$ can equivalently be expressed as

$$
\mathbf{A C}_{k} \mathbf{B}^{\prime}=\mathbf{A} \mathbf{L} \mathbf{L}^{-1} \mathbf{C}_{k} \mathbf{B}^{\prime}=\mathbf{A} \mathbf{L} \mathbf{C}_{k} \mathbf{L}^{-1} \mathbf{B}^{\prime}=(\mathbf{A L}) \mathbf{C}_{k}\left(\mathbf{B L} \mathbf{L}^{-1}\right)^{\prime}
$$

which shows that $\mathbf{A L}$ may replace $\mathbf{A}$ when $\mathbf{B} \mathbf{L}^{-1}$ replaces $\mathbf{B}$ or $\mathbf{C} \mathbf{L}^{-1}$ replaces $\mathbf{C}$. Joint simultaneous permutations of columns of $\mathbf{A}$ and $\mathbf{B}$ and diagonal elements of $\mathbf{C}_{k}, k=1, \ldots, K$, are allowed because they do not affect the matrix multiplication involved. However, apart from rescaling and permuting columns of $\mathbf{A}, \mathbf{B}$ and $\mathbf{C}$, there is no transformational freedom in $\mathrm{CP}$ when certain mild conditions are satisfied. This is why it is said that CP has the property of essential uniqueness.

What the mild conditions are will not be discussed here. For historical references and recent developments, see Harshman (1972), Kruskal (1977, 1989), Sidiropoulos and Bro (2000), Ten Berge and Sidiropoulos (2002), Jiang and Sidiropoulos (2004), De Lathauwer (2006), Stegeman and Ten Berge (2006), Stegeman, Ten Berge, and De Lathauwer (2006), Stegeman and Sidiropoulos (2007), Stegeman (2009), and Ten Berge and Tendeiro (2009).

Carroll and Chang have proposed CP because they needed it for a special application involving symmetric slices. That is, they needed to fit the constrained CP model

$$
\mathbf{X}_{k}=\mathbf{A} \mathbf{D}_{k} \mathbf{A}^{\prime}+\mathbf{E}_{k}
$$

where $\mathbf{X}_{k}=\mathbf{X}_{k}^{\prime}, k=1, \ldots, K$, in the scalar product version of a method called Indscal. Clearly, this is equivalent to (1) with the constraint $\mathbf{A}=\mathbf{B}$ added. The idea was that running the unconstrained version of $\mathbf{C P}$ would converge to a solution with $\mathbf{A}$ and $\mathbf{B}$ equal up to rescaling (e.g., see Bennani Dosse \& Ten Berge, 2008, and Ten Berge, Stegeman \& Bennani Dosse, 2009). Again, Indscal has the property of essential uniqueness just like $\mathrm{CP}$.

\section{Tucker Three-Way PCA}

Tucker three-way PCA (3PCA) has been proposed by Tucker (1966). Kroonenberg and De Leeuw (1980) have developed an alternating least-squares algorithm to fit 3PCA. It yields matrices $\mathbf{A}, \mathbf{B}$, and $\mathbf{C}$, with $P, Q$, and $R$ columns, respectively, and a $P \times Q \times R$ core array $\underline{\mathbf{G}}$ such 
that the sum of squares of elements of $\underline{\mathbf{E}}$ is minimized in the decomposition

$$
\underline{\mathbf{X}}=\sum_{p=1}^{P} \sum_{q=1}^{Q} \sum_{r=1}^{R} g_{p q r}\left(\mathbf{a}_{p} \circ \mathbf{b}_{q} \circ \mathbf{c}_{r}\right)+\underline{\mathbf{E}},
$$

where $\mathbf{a}_{p} \circ \mathbf{b}_{q} \circ \mathbf{c}_{r}$ is a so-called outer product of three vectors. It is the three-way array holding slices $\mathbf{a}_{p} \mathbf{b}_{q}^{\prime}$, multiplied in the third direction by the elements of $\mathbf{c}_{r}$. Such arrays are called rankone arrays, because they are the three-way counterparts of rank-one matrices.

It is important to note that $\mathrm{CP}$ is a constrained version of 3PCA, when $P=Q=R$ and the core array is unit superdiagonal: $g_{p q r}=0$ unless $p=q=r$, in which case $g_{p q r}=1$. Hence, the $i$ th frontal slice of $\underline{\mathbf{G}}$ is $\mathbf{e}_{i} \mathbf{e}_{i}^{\prime}$, where $\mathbf{e}_{i}$ is column $i$ of the identity matrix $\mathbf{I}$.

Unlike CP, 3PCA has no uniqueness properties. This can be seen from the matrix form $\mathbf{X}$ of 3PCA, which is decomposed as

$$
\mathbf{X}=\left[\mathbf{X}_{1}|\ldots| \mathbf{X}_{K}\right]=\mathbf{A G}\left(\mathbf{C}^{\prime} \otimes \mathbf{B}^{\prime}\right)+\left[\mathbf{E}_{1}|\ldots| \mathbf{X}_{K}\right]
$$

where $\mathbf{G}=\left[\mathbf{G}_{1}|\ldots| \mathbf{G}_{R}\right]$ is the matrix form of the core array $\underline{\mathbf{G}}$. When $\mathbf{S}, \mathbf{T}$, and $\mathbf{U}$ are nonsingular matrices, we may equivalently write the fitted part $\mathbf{A G}\left(\mathbf{C}^{\prime} \otimes \mathbf{B}^{\prime}\right)$ as $\mathbf{A}\left(\mathbf{S}^{\prime}\right)^{-1} \mathbf{S}^{\prime} \mathbf{G}\left(\mathbf{C}^{\prime} \otimes \mathbf{B}^{\prime}\right)$, and similar transformations can be applied to $\mathbf{B}$ and $\mathbf{C}$, yielding new component matrices $\mathbf{A}\left(\mathbf{S}^{\prime}\right)^{-1}$, $\mathbf{B}\left(\mathbf{T}^{\prime}\right)^{-1}$ and $\mathbf{C}\left(\mathbf{U}^{\prime}\right)^{-1}$ associated with a new core array $\mathbf{S}^{\prime} \mathbf{G}(\mathbf{U} \otimes \mathbf{T})$. This invariance property implies that there is no uniqueness in 3PCA. That is, without loss of fit, we may pre-multiply all frontal slices of $\underline{\mathbf{G}}$ by $\mathbf{S}^{\prime}$, post-multiply them by $\mathbf{T}$ and 'mix' them (take linear combinations) by $\mathbf{U}$, provided that we apply the inverse transformations to $\mathbf{A}, \mathbf{B}$, and $\mathbf{C}$, respectively. These transformations are called Tucker transformations (Rocci \& Ten Berge, 2002).

\section{Hybrid Models}

When comparing (3) and (4), it can be seen that 3PCA uses all outer products that can be formed from columns of $\mathbf{A}, \mathbf{B}$, and $\mathbf{C}$, whereas $\mathrm{CP}$ uses only those composed of the corresponding columns of $\mathbf{A}, \mathbf{B}$, and $\mathbf{C}$. In certain applications of 3PCA, however, some of the possible combinations are hypothesized to play no role. In these cases, 3PCA is constrained to have certain elements of the core fixed to zero. This creates hybrid models that are "in between" 3PCA and CP. For instance, when it is hypothesized that the core must be a $3 \times 3 \times 3$ array of the (matricized) form

$$
\mathbf{G}=\left[\mathbf{G}_{1}\left|\mathbf{G}_{2}\right| \mathbf{G}_{3}\right]=\left[\begin{array}{ccc|ccc|ccc}
a & 0 & 0 & 0 & 0 & 1 & 0 & 1 & 0 \\
0 & 0 & 1 & 0 & b & 0 & 1 & 0 & 0 \\
0 & 1 & 0 & 1 & 0 & 0 & 0 & 0 & c
\end{array}\right]
$$

for certain scalars $a, b$, and $c$, we are in between 3PCA (no zeros) and the CP core

$$
\mathbf{H}=\left[\mathbf{H}_{1}\left|\mathbf{H}_{2}\right| \mathbf{H}_{3}\right]=\left[\begin{array}{ccc|ccc|ccc}
1 & 0 & 0 & 0 & 0 & 0 & 0 & 0 & 0 \\
0 & 0 & 0 & 0 & 1 & 0 & 0 & 0 & 0 \\
0 & 0 & 0 & 0 & 0 & 0 & 0 & 0 & 1
\end{array}\right]
$$

which has 24 zeros. Surprisingly, the essential uniqueness associated with the CP core (7) is not lost when six constraints are relaxed as in (6). That is, when Tucker transformations are sought to transform $\mathbf{A}, \mathbf{B}$, and $\mathbf{C}$ without changing the core (6), the only solutions that appear almost surely involve merely permutations and joint rescaling of their columns (Kiers, Ten Berge \& Rocci, 1997). Uniqueness is highly desirable for hybrid models. It is even more important to 
ascertain whether or not the model is trivial. That is, when the hypothesized core is of a form that can be attained by merely applying Tucker transformations to any arbitrary core of the given format, the hypothesis is necessarily true, and therefore trivial. For example, Rocci and Ten Berge (2002) have shown how almost any pair of $3 \times 3$ matrices can be changed to the form

$$
\left[\begin{array}{lll|lll}
1 & 0 & 0 & 0 & 0 & 0 \\
0 & 1 & 0 & 0 & 0 & x \\
0 & 0 & 1 & 0 & y & 0
\end{array}\right]
$$

by using Tucker transformations. It follows that there is no point in hypothesizing that the 13 zeros of this core are due to the particular nature of one's data. The hypothesis will be trivial and instead of having a model, we are looking at a tautology.

Questions of uniqueness and triviality can be dealt with on an ad hoc basis, by examining the specific constrained core at hand. Preferably, however, answers should come from general principles. Such principles exist in the form of either simplicity results or typical rank results. These will be explained next.

\section{Transforming Arrays to Maximal Simplicity}

Kiers (1998) has developed the Simplimax method, which uses the transformational indeterminacy, explained below (5), to create large number of zeros in an array by an iterative procedure. Experiences with this method were promising enough to start a search for closed-form transformations to simplicity.

The basic idea of simplicity transformations is well known from matrix algebra. For instance, multiplying an $n \times n$ matrix by its inverse yields as few as $n$ nonzero elements. Multiplying any matrix by its singular vector matrices on both sides yields a matrix with all elements zero except those that are the singular values. For matrix pairs that can be diagonalized simultaneously, again a vast majority of the elements will vanish. The question is to what extent such procedures generalize to three-way arrays.

A first example is obvious. Consider a $5 \times 2 \times 2$ array with slices $\mathbf{X}_{1}$ and $\mathbf{X}_{2}$. This array can be transformed to a form where

$$
\mathbf{S}^{\prime} \mathbf{X}_{1}=\left[\begin{array}{ll}
1 & 0 \\
0 & 1 \\
0 & 0 \\
0 & 0 \\
0 & 0
\end{array}\right] \quad \text { and } \quad \mathbf{S}^{\prime} \mathbf{X}_{2}=\left[\begin{array}{ll}
0 & 0 \\
0 & 0 \\
0 & 0 \\
1 & 0 \\
0 & 1
\end{array}\right] .
$$

The solution is trivially easy to find. Construct a $5 \times 5$ matrix holding $\mathbf{X}_{1}$ as first two columns, $\mathbf{X}_{2}$ as last two columns, and a random third column in between. This matrix usually has an inverse. Taking $\mathbf{S}^{\prime}$ as that inverse gives the desired result. Unfortunately, this example has no applications in 3PCA because a core of format $5 \times 2 \times 2$ is a case of overfactoring: The same fit can be attained by using a core of format $4 \times 2 \times 2$ (Tucker, 1966).

A slightly more difficult case is that of a $3 \times 2 \times 2$ core. Again, we want to attain the pattern of "first columns of the identity matrix in slice 1 and last columns in slice 2", but now we also need to post-multiply the slices by a nonsingular $\mathbf{T}$. That is, we want to find nonsingular matrices $\mathbf{S}$ and $\mathbf{T}$ such that

$$
\mathbf{S}^{\prime} \mathbf{X}_{1} \mathbf{T}=\left[\begin{array}{ll}
1 & 0 \\
0 & 1 \\
0 & 0
\end{array}\right] \quad \text { and } \quad \mathbf{S}^{\prime} \mathbf{X}_{2} \mathbf{T}=\left[\begin{array}{ll}
0 & 0 \\
1 & 0 \\
0 & 1
\end{array}\right]
$$


Let $\mathbf{S}=\left[\mathbf{s}_{1}\left|\mathbf{s}_{2}\right| \mathbf{s}_{3}\right]$. Noting that the rows of $\mathbf{S}^{\prime} \mathbf{X}_{1} \mathbf{T}$ also appear as rows of $\mathbf{S}^{\prime} \mathbf{X}_{2} \mathbf{T}$, albeit in different places, we need to solve the four equations

$$
\begin{aligned}
\mathbf{s}_{1}^{\prime} \mathbf{X}_{1} \mathbf{T} & =\mathbf{s}_{2}^{\prime} \mathbf{X}_{2} \mathbf{T}, \\
\mathbf{s}_{2}^{\prime} \mathbf{X}_{1} \mathbf{T} & =\mathbf{s}_{3}^{\prime} \mathbf{X}_{2} \mathbf{T}, \\
\mathbf{s}_{3}^{\prime} \mathbf{X}_{1} \mathbf{T} & =\mathbf{0}^{\prime}, \\
\mathbf{s}_{1}^{\prime} \mathbf{X}_{2} \mathbf{T} & =\mathbf{0}^{\prime} .
\end{aligned}
$$

Post-multiplying these equations by $\mathbf{T}^{-1}$ will remove $\mathbf{T}$ from the equations. Upon stacking $\mathbf{s}_{1}$, $\mathbf{s}_{2}$, and $\mathbf{s}_{3}$ in a column vector $\mathbf{s}$, it can be seen that the four equations have a solution if and only if $\mathbf{s}$ is orthogonal to the columns of the $9 \times 8$ matrix

$$
\mathbf{W}=\left[\begin{array}{c|c|c|c}
\mathbf{X}_{1} & \mathbf{O} & \mathbf{O} & \mathbf{X}_{2} \\
\hline-\mathbf{X}_{2} & \mathbf{X}_{1} & \mathbf{O} & \mathbf{O} \\
\hline \mathbf{O} & -\mathbf{X}_{2} & \mathbf{X}_{1} & \mathbf{O}
\end{array}\right]
$$

Upon picking $\mathbf{s}$ orthogonal to column space of $\mathbf{W}$, and rearranging it back into a matrix, we have a $3 \times 3$ matrix $\mathbf{S}$ that is almost surely nonsingular (with probability 1, that is, when $\mathbf{X}_{1}$ and $\mathbf{X}_{2}$ are randomly sampled from a continuous distribution). Once $\mathbf{S}$ has been found, $\mathbf{T}$ follows as the inverse of the matrix holding the first two rows of $\mathbf{S}^{\prime} \mathbf{X}_{1}$. It may be noted that this transformation uses pre-multiplication of the slices by $\mathbf{S}^{\prime}$ and post-multiplication by $\mathbf{T}$, but not the slice mix transformation $\mathbf{U}$. This implies that the transformation that yields (10) is not unique.

The case just treated is not an isolated result. In fact, for all $I \times J \times 2$ arrays with $I>J$, the matrix $\mathbf{W}$ is tall and a vector $\mathbf{s}$ orthogonal to its column space exists. The resulting $\mathbf{S}$ will be nonsingular almost surely. The transformed array will have slices holding the first $J$ columns and the last $J$ columns of $\mathbf{I}_{I}$, respectively (Ten Berge \& Kiers, 1999). However, when $I=J$ this does not work and a different approach is needed (Rocci \& Ten Berge, 2002).

It is not difficult to verify that the simplicity result, yielding $2 J$ nonzero elements and $2 I J-2 J$ zeros, is also a maximal simplicity result. That is, attaining more zeros in a $I \times J \times 2$ array with $I>J$ is almost surely impossible. The proof can be found in Rocci and Ten Berge (2002). They also show that a simplicity transformation for $I \times J \times K$ arrays with $I=J K-1$ by Murakami, Ten Berge, and Kiers (1998) has maximum simplicity. More recently, Tendeiro, Ten Berge, and Kiers (2009) have given simplicity results for partially symmetric arrays. For example, when $4 \times 3 \times 3$ arrays have symmetric slices, they almost surely admit a maximally simple form

$$
\left[\begin{array}{ccc|ccc|ccc|ccc}
1 & 0 & 0 & 1 & 0 & 0 & 0 & 1 & 0 & 0 & 0 & 1 \\
0 & \alpha & 0 & 0 & 0 & 0 & 1 & 0 & 0 & 0 & 0 & 0 \\
0 & 0 & 0 & 0 & 0 & \pm \alpha & 0 & 0 & 0 & 1 & 0 & 0
\end{array}\right]
$$

for some scalar $\alpha$.

At this point, we may revert to the triviality question in hybrid models. When a hypothesized core array contains more zeros than is admitted by a maximum simplicity result, the hypothesis is non-trivial. For instance, when a $5 \times 4 \times 2$ array would be hypothesized to have fewer than eight nonzero elements, that hypothesis would be non-trivial. Unfortunately, although some cases have been solved, for most array formats the maximum simplicity is still unknown. However, additional general rules for deciding about triviality can be obtained from rank results of threeway arrays. These will be treated in the remainder of this paper. 


\section{Typical Rank of Three-way Arrays}

The concept of three-way rank goes back to Hitchcock (1927b). Just like the concept of a rank-one matrix (outer product of two vectors) generalizes smoothly to the concept of rank-one arrays (outer product of three vectors), the concept of matrix rank (smallest number of rank-one matrices that have the matrix as their sum) generalizes smoothly to the concept of three-way rank: It is the smallest number of rank-one arrays that have the array as their sum. In the present paper, we are dealing with the rank over the real number field, exclusively.

For matrices, the maximal rank coincides with the "typical" rank, which is the rank that arises almost surely when the matrix entries are randomly sampled from a continuous distribution. So a random $I \times J$ matrix, $I \geq J$, will have rank $J$ almost surely. Kruskal (1983) pointed out that a three-way array format may have a typical rank that differs from its maximal rank. In addition, he discussed cases where an array format has two typical rank values. For instance, a $2 \times 2 \times 2$ array has rank 2 with probability $p, 0<p<1$, and rank 3 with probability $1-p$, almost surely. A $4 \times 4 \times 2$ array has typical rank $\{4,5\}$ and maximal rank 6 (Thijsse, 1994, also see Ten Berge, 2000). This means that, although arrays that have rank 6 can be constructed, their set has Lebesgue measure zero.

The concepts of rank and typical rank have received considerable attention in three-way analysis, because the rank coincides with the smallest number of components that yield perfect fit in CP. An important fact about rank is its invariance over Tucker transformations. When frontal slices are pre-multiplied by $\mathbf{S}^{\prime}$, post-multiplied by $\mathbf{T}$, and mixed by $\mathbf{U}$, the outer products of the form $\mathbf{a}_{j} \circ \mathbf{b}_{j} \circ \mathbf{c}_{j}$ change to $\mathbf{S}^{\prime} \mathbf{a}_{j} \circ \mathbf{T} \mathbf{b}_{j} \circ \mathbf{U} \mathbf{c}_{j}$ but the number of these rank-one arrays needed to account for the transformed array remains the same. Therefore, Tucker transformations may be used to simplify arrays before examining their typical ranks. For example, consider the $3 \times 2 \times 2$ array treated above, which admits a simplified form

$$
\mathbf{X}_{1}=\left[\begin{array}{ll}
1 & 0 \\
0 & 1 \\
0 & 0
\end{array}\right] \quad \text { and } \quad \mathbf{X}_{2}=\left[\begin{array}{ll}
0 & 0 \\
1 & 0 \\
0 & 1
\end{array}\right]
$$

Pre-multiplying these slices by the Vandermonde matrix

$$
\mathbf{V}=\left[\begin{array}{lll}
1 & 1 & 1 \\
1 & 2 & 4 \\
1 & 3 & 9
\end{array}\right]
$$

yields new slices

$$
\mathbf{V X}_{1}=\left[\begin{array}{ll}
1 & 1 \\
1 & 2 \\
1 & 3
\end{array}\right] \text { and } \quad \mathbf{V X}_{2}=\left[\begin{array}{ll}
1 & 1 \\
2 & 4 \\
3 & 9
\end{array}\right]
$$

holding the first two and the last two columns of $\mathbf{V}$, respectively. At this point, it can be concluded that the array has rank 3 . That is, its rank is at least 3 because the four columns span the three dimensional space so the component matrix $\mathbf{A}$ of $\mathbf{C P}$, see (1), must have at least three columns. Its rank is also at most 3, because each row of $\mathbf{V} \mathbf{X}_{1}$ is proportional to its counterpart in $\mathbf{V} \mathbf{X}_{2}$, so each horizontal slice of the array is a rank-one array. Because the transformation (13) is possible almost surely, $3 \times 2 \times 2$ arrays have typical rank 3 . This result holds more generally for all $I \times J \times 2$ arrays with $2 J>I>J$. Their typical rank is $I$ (Ten Berge \& Kiers, 1999).

The transformation to maximal simplicity demonstrated above served as a stepping stone for the Vandermonde transformation (14). The latter rendered the typical rank visible to the naked eye, by enforcing row-wise proportionality. This principle also applies in many cases where 
TABLE 1.

Typical rank values for fully random arrays with $I \geq J \geq K$.

\begin{tabular}{|c|c|c|c|c|c|c|c|c|c|c|}
\hline & \multicolumn{3}{|c|}{$K=2$} & \multicolumn{3}{|c|}{$K=3$} & \multicolumn{4}{|c|}{$K=4$} \\
\hline$I=2$ & $\begin{array}{l}J=2 \\
\end{array}$ & $J=3$ & $J=4$ & $J=3$ & $J=4$ & $J=5$ & $J=4$ & $J=5$ & $J=6$ & $J=7$ \\
\hline$I=3$ & 3 & $\{3,4\}$ & & 5 & & & & & & \\
\hline$I=4$ & 4 & 4 & $\{4,5\}$ & $?$ & $\geq 6$ & & $\geq 6$ & & & \\
\hline$I=5$ & 4 & 5 & 5 & $\{5,6]$ & $?$ & $\geq 7$ & $?$ & $\geq 7$ & & \\
\hline$I=6$ & 4 & 6 & 6 & 6 & $?$ & $?$ & $?$ & $?$ & $\geq 8$ & \\
\hline$I=7$ & 4 & 6 & 7 & 7 & $\{7,8, ?\}$ & $?$ & $?$ & $?$ & $?$ & $\geq 9$ \\
\hline$I=8$ & 4 & 6 & 8 & 8 & $\{8,9\}$ & $?$ & $?$ & $?$ & $?$ & $?$ \\
\hline$I=9$ & 4 & 6 & 8 & 9 & 9 & $\{9,10\}$ & $?$ & $?$ & $?$ & $?$ \\
\hline$I=10$ & 4 & 6 & 8 & 9 & 10 & 10 & $\{10, ?\}$ & $?$ & $?$ & $?$ \\
\hline$I=11$ & 4 & 6 & 8 & 9 & 11 & 11 & $\{?\}$ & $?$ & $?$ & \\
\hline$I=12$ & 4 & 6 & 8 & 9 & 12 & 12 & $\{12,13\}$ & $?$ & $?$ & \\
\hline$I=13$ & 4 & 6 & 8 & 9 & 12 & 13 & 13 & $\{?\}$ & $?$ & \\
\hline$I=14$ & 4 & 6 & 8 & 9 & 12 & 14 & 14 & $?$ & $?$ & \\
\hline$I=15$ & 4 & 6 & 8 & 9 & 12 & 15 & 15 & 15 & $?$ & \\
\hline$I=16$ & 4 & 6 & 8 & 9 & 12 & 15 & 16 & 16 & $\{?\}$ & \\
\hline$I=17$ & 4 & 6 & 8 & 9 & 12 & 15 & 16 & 17 & $?$ & \\
\hline$I=18$ & 4 & 6 & 8 & 9 & 12 & 15 & 16 & 18 & $\{18, ?\}$ & \\
\hline$I=19$ & 4 & 6 & 8 & 9 & 12 & 15 & 16 & 19 & 19 & $\{?\}$ \\
\hline$I=20$ & 4 & 6 & 8 & 9 & 12 & 15 & 16 & 20 & 20 & \\
\hline$I=21$ & 4 & 6 & 8 & 9 & 12 & 15 & 16 & 20 & 21 & 21 \\
\hline$I=22$ & 4 & 6 & 8 & 9 & 12 & 15 & 16 & 20 & 22 & 22 \\
\hline
\end{tabular}

no simplicity result is available. Specifically, for so-called "tall arrays" of format $I \times J \times K$ with $J K>I>J K-J$, the Vandermonde matrix can be used to attain row-wise proportionality without starting from simplicity. Such arrays also have rank $I$ (Ten Berge, 2000).

\section{An Overview of Typical Rank Results}

Various authors have given tables of typical rank results. These tables focus on solved cases. Table 1 contains an update of these tables and also specifies open problems, in the form of question marks. The table clarifies the classification of $I \times J \times K$ array formats with $I \geq J \geq K$ as very tall (uncolored) when $I \geq J K$, as tall (green) when $J K>I>J K-J$, and as compact otherwise.

The uncolored numbers in the south-west corner of the table refer to the very tall arrays. Their typical rank is trivially $J K$, because every $J \times K$ matrix can be written as the sum of at most $J K$ rank-one matrices. The green cells refer to tall arrays. Their typical rank is $I$, as has been explained above.

The yellow cells refer to the tallest among the compact arrays. For $K=2$, their typical ranks are $\{I, I+1\}$ (see Kruskal, 1989; Ten Berge, 1991, and Ten Berge and Kiers, 1999). For $K>2$, however, we have an incomplete set of results (Ten Berge, 2000). When $J$ is odd, the typical rank is $I$, and when $J$ is even, the typical rank is either $I$ or it is $\{I, I+1\}$, a ramification that seems to be missing in Table 3.3 of Kolda \& Brader (2009). The first case with $J$ even is the $8 \times 4 \times 3$ case. Sumi, Sakata, and Miyazaki (2010) have shown that arrays with rank above 8 arise with positive probability, so the typical rank is $\{8,9\}$. The next case is the $12 \times 4 \times 4$ case, which was open in Ten Berge (2000) but can now be considered as closed. Friedland (2010) has shown that such arrays have a rank above 12 with positive probability, so the $12 \times 4 \times 4$ array has typical rank $\{12,13\}$. For larger array formats like $18 \times 6 \times 4$ and beyond, it can only be conjectured that the typical rank is $\{I, I+1\}$, when $J$ is even. 
TABLE 2 .

Typical ranks when slices are symmetric

\begin{tabular}{c|ccc}
\hline & $J=K=2$ & $J=K=3$ & $J=K=4$ \\
\hline$I=2$ & $\{2,3\}$ & $(3,4)$ & $\{4,5\}$ \\
$I=3$ & 3 & 4 & $\geq 6$ \\
$I=4$ & 3 & $\{4,5\}$ & $?$ \\
$I=5$ & 3 & $\{5,6\}$ & $?$ \\
$I=6$ & 3 & 6 & $?$ \\
$I=7$ & 3 & 6 & $?$ \\
$I=8$ & 3 & 6 & $\{8,9\}$ \\
$I=9$ & 3 & 6 & $\{9,10\}$ \\
$I=10$ & 3 & 6 & 10 \\
\hline
\end{tabular}

The pink cells in Table 1 refer to miscellaneous results. The $3 \times 3 \times 3$ array was shown to have typical rank 5 by Ten Berge and Stegeman (2006). The $5 \times 3 \times 3$ array was shown to have typical rank $\{5,6\}$ in Ten Berge (2004), where a simplicity transformation of the Ten Berge and Kiers (1999) type was applied to the first two slices to make the problem easier to handle. Cases where slices are $I \times I$ or $J \times J$ were treated in Ten Berge and Stegeman (2006), who showed that their typical rank values are at least $I+2$ or $J+2$, respectively, when $K>2$. Finally, the cell $11 \times 4 \times 4$ is also pink. For this cell, Friedland (2010) has shown that there must be at least two typical rank values.

The grey cells refer to so-called minimal system cases (Choulakian, 2010). By generating random arrays and evaluating roots of a certain polynomial, Choulakian found cases of rank $I$, and rank higher than $I$ with positive probability. However, because it is not known if a typical rank higher than $I+1$ is impossible, these results only give lower bounds to typical rank. Only for the $9 \times 5 \times 3$ case do we have the full picture because a typical rank above 10 would exceed that of the $10 \times 5 \times 3$ array, which is impossible. For cases larger than $10 \times 4 \times 4$ the computational burden has so far been prohibitive.

All remaining cells in Table 1 have question marks. This does not mean that there is no information on these cases. Firstly, by interpolation, the lower bounds in pink cells also apply to all cells below them, because adding slices (increasing $I$ for fixed $J$ and $K$ ) cannot reduce rank. Secondly, Comon, Ten Berge, De Lathauwer, and Castaing (2009) have reported lower bounds to typical rank based on numerical computations that could replace the question marks. All bounds evaluated so far by their method have been in full agreement with the known results of Table 1 .

It is well known that typical ranks change when the slices of an array are symmetric. Table 2 summarizes what is known about the typical rank of arrays holding $I$ symmetric slices of order $J \times J$. As in Table 1, the uncolored numbers refer to trivial cases, where the number of slices $I$ equals or exceeds the number of rank-one matrices needed to span the space of symmetric $J \times J$ matrices (Rocci \& Ten Berge, 1994). The green cells refer to cases treated by Ten Berge, Sidiropoulos and Rocci (2004). The pink cells again refer to miscellaneous results. The $3 \times 4 \times 4$ array has a typical rank of at least 6 (Ten Berge $\&$ Stegeman, 2006). The $8 \times 4 \times 4$ and $9 \times 4 \times 4$ cases have been solved by Ten Berge, Stegeman and Bennani Dosse (2009), who took advantage of simplicity transformations by Tendeiro, Ten Berge and Kiers (2009). These transformations, incidentally, also greatly simplify the proofs of some of the results of Ten Berge, Sidiropoulos, and Rocci (2004). Again, the question marks can be replaced by interpolation and by using lower bounds of Comon et al. (2009). 


\section{Discussion}

Simplicity transformations have been very useful in determining typical rank results. Typical rank is fundamental to the study of three-way decompositions. However, it also has direct applications in hybrid models. Ten Berge and Smilde (2002) discussed a 3PCA study of Gurden, Westerhuis, Bijlsma, and Smilde (2001), which involved a constrained $5 \times 5 \times 3$ core array containing no more than five nonzero elements. Ten Berge and Smilde showed that this core, which has rank 5, is non-trivial, because the typical rank of $5 \times 5 \times 3$ arrays is at least 6 , which means that this core cannot be obtained almost surely from Tucker transformations of any arbitrary core. It can be seen from Table 1 that the typical rank is at least 7, but 6 was already enough to decide on triviality of this particular core.

Tables 1 and 2 reflect classes of typical rank problems that have been solved, but also reveal which cases need further treatment. A case where a solution is conspicuously absent is that of the $4 \times 3 \times 3$ arrays. It can be shown that rank 5 has positive probability and that the typical rank is below 7, but other than that nothing has been achieved.

\section{References}

Bennani Dosse, M., \& Ten Berge, J.M.F. (2008). The assumption of proportional components when Candecomp is applied to symmetric matrices in the context of Indscal. Psychometrika, 73, 303-307.

Carroll, J.D., \& Chang, J.J. (1970). Analysis of individual differences in multidimensional scaling via an $n$-way generalization of Eckart-Young decomposition. Psychometrika, 35, 283-319.

Choulakian, V. (2010). Some numerical results on the rank of generic three-way arrays over $\Re$. SIAM Journal on Matrix Analysis and Applications, 31, 1541-1551.

Comon, P., Ten Berge, J.M.F., De Lathauwer, L., \& Castaing, J. (2009). Generic and typical ranks of multiway arrays. Linear Algebra \& Applications, 430, 2997-3007.

De Lathauwer, L. (2006). A link between the canonical decomposition in multilinear algebra and simultaneous matrix diagonalization. SIAM Journal on Matrix Analysis and Applications, 28, 642-666.

Gurden, S.P., Westerhuis, J.A., Bijlsma, S., \& Smilde, A.K. (2001). Modeling of spectroscopic batch process data using grey models to incorporate external information. Journal of Chemometrics, 15, 101-121.

Friedland, S. (2010). On the generic and typical rank of 3-tensors. arXiv:0805.3777v4.

Harshman, R.A. (1970). Foundations of the PARAFAC procedure: Models and conditions for an "explanatory" multimode factor analysis. UCLA Working Papers in Phonetics, 16, 1-84.

Harshman, R.A. (1972). Determination and proof of minimum uniqueness conditions for PARAFAC1. UCLA Working Papers in Phonetics, 16, 1-84.

Hitchcock, F.L. (1927a). The expression of a tensor or a polyadic as a sum of products. Journal of Mathematical Physics, 6, 164-189.

Hitchcock, F.L. (1927b). Multiple invariants and generalized rank of a p-way matrix or tensor. Journal of Mathematical Physics, 7, 39-79.

Jiang, T., \& Sidiropoulos, N.D. (2004). Kruskal's permutation lemma and the identification of Candecomp/Parafac and bilinear models with constant modulus constraints. IEEE Transactions on Signal Processing, 52, 2625-2636.

Kiers, H.A.L. (1998). Three-way SIMPLIMAX for oblique rotation of the three-mode factor analysis core to simple structure. Computational Statistics \& Data Analysis, 28, 307-324.

Kiers, H.A.L., Ten Berge, J.M.F., \& Rocci, R. (1997). Uniqueness of three-mode factor models with sparse cores: The $3 \times 3 \times 3$ case. Psychometrika, 62, 349-374.

Kolda, T.G., \& Brader, B.W. (2009). Tensor decompositions and applications. SIAM Review, 51, 455-500.

Kroonenberg, P.M., \& De Leeuw, J. (1980). Principal component analysis of three-mode data by means of alternating least-squares. Psychometrika, 45, 69-97.

Kruskal, J.B. (1977). Three-way arrays: Rank and uniqueness of trilinear decompositions with applications to arithmetic complexity and statistics. Linear Algebra \& Applications, 18, 95-138.

Kruskal, J.B. (1983, unpublished). Statement of some current results about three-way arrays.

Kruskal, J.B. (1989). Rank, decomposition, and uniqueness for 3-way and $N$-way arrays. In Coppi, R., \& Bolasco, S. (Eds.) Multiway data analysis (pp. 7-18). Amsterdam: North-Holland.

Murakami, T., Ten Berge, J.M.F., \& Kiers, H.A.L. (1998). A case of extreme simplicity of the core matrix in three-mode principal component analysis. Psychometrika, 63, 255-261.

Rocci, R., \& Ten Berge, J.M.F. (1994). A simplification of a result by Zellini on the maximal rank of a symmetric three-way array. Psychometrika, 59, 377-380.

Rocci, R., \& Ten Berge, J.M.F. (2002). Transforming three-way arrays to maximal simplicity. Psychometrika, 67, 351365.

Sidiropoulos, N.D., \& Bro, R. (2000). On the uniqueness of multilinear decomposition of $N$-way arrays. Journal of Chemometrics, 14, 229-239. 
Stegeman, A.W. (2009). On uniqueness conditions for Candecomp/Parafac and Indscal with full column rank in one mode. Linear Algebra \& Applications, 431, 211-227.

Stegeman, A.W., \& Ten Berge, J.M.F. (2006). Kruskal's condition for uniqueness in Candecomp/Parafac when ranks and $k$-ranks coincide. Computational Statistics \& Data Analysis, 50, 210-220.

Stegeman, A., Ten Berge, J.M.F., \& De Lathauwer, L. (2006). Sufficient conditions for uniqueness in Candecomp/Parafac and Indscal with random component matrices. Psychometrika, 71, 219-229.

Stegeman, A., \& Sidiropoulos, N.D. (2007). On Kruskal's uniqueness condition for the Candecomp/Parafac decomposition. Linear Algebra \& Applications, 420, 540-552.

Sumi, T., Sakata, T., \& Miyazaki, M. (2010). Typical ranks for $m \times n \times(m-1) n$ tensors with $m \leq n$. Preprint, retrieved from http://polygon.aid.design.kyushu-u.ac.jp/ sumi/myarticles.html, October 14, 2010.

Ten Berge, J.M.F. (1991). Kruskal's polynomial for $2 \times 2 \times 2$ arrays and a generalization to $2 \times n \times n$ arrays. Psychometrika, 56, 631-636.

Ten Berge, J.M.F. (2000). The typical rank of tall three-way arrays. Psychometrika, 65, 525-532.

Ten Berge, J.M.F. (2004). Partial uniqueness in CANDECOMP/PARAFAC. Journal of Chemometrics, 18, $12-16$.

Ten Berge, J.M.F., \& Kiers, H.A.L. (1999). Simplicity of core arrays in three-way principal component analysis and the typical rank of $P \times Q \times 2$ arrays. Linear Algebra \& Applications, 294, 169-179.

Ten Berge, J.M.F., \& Sidiropoulos, N.D. (2002). Some new results on uniqueness in Candecomp/Parafac. Psychometrika, 67, 399-409.

Ten Berge, J.M.F., Sidiropoulos, N.D., \& Rocci, R. (2004). Typical rank and Indscal dimensionality for symmetric threeway arrays of order $I \times 2 \times 2$ or $I \times 3 \times 3$. Linear Algebra \& Applications, 388, 363-377.

Ten Berge, J.M.F., \& Smilde, A.K. (2002). Non-triviality and identification of a constrained Tucker3 analysis. Journal of Chemometrics, 16, 609-612.

Ten Berge, J.M.F., \& Stegeman, A. (2006). Symmetry transformations for square sliced three-way arrays, with applications to their typical rank. Linear Algebra \& Applications, 418, 215-224.

Ten Berge, J.M.F., Stegeman, A., \& Bennani Dosse, M. (2009). The Carroll-Chang conjecture of equal Indscal components when Candecomp/Parafac gives perfect fit. Linear Algebra \& Applications, 430, 818-829.

Ten Berge, J.M.F., \& Tendeiro, J.N. (2009). The link between sufficient conditions by Harshman and by Kruskal for uniqueness in Candecomp/Parafac. Journal of Chemometrics, 23, 321-323.

Tendeiro, J.N., Ten Berge, J.M.F., \& Kiers, H.A.L. (2009). Simplicity transformations for three-way arrays with symmetric slices, and applications to Tucker-3 models with sparse core arrays. Linear Algebra \& Applications, 430, 924-940.

Thijsse, G.P.A. (1994). Simultaneous diagonal forms for pairs of matrices (Report 9450/B). Econometric Institute. Erasmus University, Rotterdam.

Tucker, L.R. (1966). Some mathematical notes on three-mode factor analysis. Psychometrika, 31, 279-311.

Published Online Date: 5 JAN 2011 